IV.

\title{
Case Illustrating the Effect of Pregnancy on Alopecia Areata.
}

By G. G. Stopford Taylor, M.D., and R. W. Mackenna, M.A., M.B., Liverpool.

IT is a matter of elementary knowledge that pregnancy produces changes in other organs of the body besides those more intimately associated with gestation. The skin partakes in these alterations, and the obstetrician is probably more familiar than the dermatologist with the pigmentary changes induced in the integument by the gravid state. The linea nigra, the darkening of the areola, the development of the secondary areola, the proliferation and hypertrophy of the sweat and sebaceous glands-of which the formation of Montgomery's follicles is a special instance,- the improvement in the texture and the quantity of the hair, and the deposition of a large amount of subcutaneous fat, especially over the buttocks and in the mammary regions, are all indications that the skin and its subjacent structures are in a state of abnormal functional activity. Occasionally this activity brings about pathological changes such as pigmentary deposits in exposed parts of the body like the face, or the removal of pigment from one area of the skin to another giving rise to leucoderma.

The following case, which presents features of great interest both for the obstetric physician and the dermatologist, was recently seen at our clinique :

CASE. Mrs. D., age 32. When the patient was between six and seven years of age she had a first attack of Alopecia areata consisting of some six or eight bald patches on the scalp. There had been no previous illness to occasion this loss of hair, and otherwise she was in good health. The attack persisted for about a year, recovery taking place under local and general treatment. At about the age of $14 \frac{1}{2}$ there was another attack, confined to the scalp, which lasted about four years. The hair on the eyebrows, and the eyelashes were spared, but on the scalp there was a progressive loss until complete baldness was established.

Menstruation began at the age of $15 \frac{1}{4}$ years, but though the patient's body underwent the usual structural modifications characteristic of puberty, such as the development of hair on the pubes and in the axillæ, the hair did not return to the scalp until between her eighteenth and nineteenth year. There was no further attack till after marriage, which took place at the age of 25. During her first 
pregnancy the patient noticed that her hair grew very thick and strong. When the pregnancy terminated, and during lactation, the hair began to fall out and continued to do so till there was complete baldness. This attack lasted for two and a half years, when the patient again became pregnant. A few weeks after conception the hair began to grow again, and by the time of parturition she had a plentiful supply. Since the pregnancy terminated, about two years ago, there has been universal alopecia, the hair having fallen from the scalp, the eyebrows, eyelids, armpits, genitalia, limbs and trunk. This is the most severe attack the patient has yet experienced, but the curious cycle of events is being repeated, for she is now again in the third month of pregnancy, and there are signs of a rapid return of hair to all the denuded areas.

During pregnancy the patient enjoys very good health, and has not noticed any special pigmentation, or felt any special nerve pains. She suckles her children for about ten months and does not feel it a strain to do so.

There are many interesting points in this case. Of recent years there has grown up a considerable body of opinion that alopecia areata is a disease of microbic origin, and various microbes have been isolated and dignified as the causes of alopecia. This case, at any rate, adds to the weight of evidence against the microbic theory.

Another point of interest in the case is the fact that when menstruation was established, though the hair follicles on the pubes and in the axillæ, which usually become active at puberty, began to develop there was no similar activity generated in the hair follicles of the scalp, which remained bald for quite three years. The case is apparently one in which there is a constitutional tendency to alopecia, and the stimulus of pregnancy, producing as it does greater activity of the sebaceous glands and an increase in the total volume of blood in the body, communicates to the hair follicles with which the sebaceous glands are associated a germinal impulse which sets up active growth. When pregnancy terminates, the sebaceous glands cease to be so active, there is a loss of subcutaneous fat, and the volume of blood returns to the normal. The activities of the skin are more quiescent, and the hair follicles apparently lack sufficient vitality to keep up a growth of hair. 